\title{
A new numerical approach for MHD laminar boundary layer flow and heat transfer of nanofluids over a moving surface in the presence of thermal radiation
}

\author{
Stanford Shateyi ${ }^{1 *}$ and Jagdish Prakash ${ }^{2}$
}

\author{
"Correspondence: \\ stanford.shateyi@univen.ac.za \\ ${ }^{1}$ Department of Mathematics, \\ University of Venda, Private Bag \\ X5050, Thohoyandou, 0950, South \\ Africa \\ Full list of author information is \\ available at the end of the article
}

\begin{abstract}
Numerical analysis has been carried out on the problem of magnetohydrodynamic boundary layer flow of a nanofluid over a moving surface in the presence of thermal radiation. The governing partial differential equations were transformed into a system of ordinary differential equations using suitable similarity transformations. The resultant ordinary equations were then solved using the spectral relaxation method. Effects of the physical parameters on the velocity, temperature and concentration profiles as well as the local skin-friction coefficient and the heat and mass transfer rates are depicted graphically and/or in tabular form.
\end{abstract}

MSC: $65 \mathrm{PXX} ; 76-\mathrm{XX}$

Keywords: MHD boundary layer flow; nanofluids; thermal radiation; moving surface

\section{Introduction}

Many engineering and industrial processes involve heat transfer by means of a flowing fluid in either laminar or turbulent regimes. A decrease in thermal resistance of heat transfer in the fluids would significantly benefit many of these applications/processes. Nanofluids have the potential to reduce thermal resistances, and industrial groups such as electronics, medical, food and manufacturing would benefit from such improved heat transfer. It is well known that conventional heat transfer fluids, such as oil, water and ethylene glycol mixture, are poor heat transfer fluids. Choi [1] introduced the technique of nanofluids by using a mixture of nanoparticles and the base fluids. The presence of the nanoparticles in the nanofluid increases the thermal conductivity and therefore substantially enhances the heat transfer characteristics of the nanofluid. Nanotechnology has been an ongoing hot topic of discussion in public health as researchers claim that nanoparticles could present possible dangers in health and environment, Mnyusiwella et al. [2]. There are several numerical studies on the modelling of natural convection heat transfer in nanofluids (Kaka and Pramuanjaroekij [3], Godson et al. [4], Olanrewaju et al. [5]). Gbadeyan et al. [6] numerically studied boundary layer flow induced in a nanofluid due to a linearly stretching sheet in the presence of thermal radiation and induced magnetic field. Khan and Aziz [7] studied natural convection flow of a nanofluid over a vertical plate with a uniform surface heat flux. Makinde and Aziz [8] investigated boundary layer flow of a nanofluid past

๑2014 Shateyi and Prakash; licensee Springer. This is an Open Access article distributed under the terms of the Creative Commons Attribution License (http://creativecommons.org/licenses/by/2.0), which permits unrestricted use, distribution, and reproduction in any medium, provided the original work is properly cited. 
a stretching sheet with a convective boundary conditions. Khan et al. [9] studied the unsteady free convection boundary layer flow of a nanofluid along a stretching sheet with thermal radiation in the presence of a magnetic field.

Thermal radiation-convection interaction problems are found in the cooling of high temperature components design where heat transfer from surfaces occurs by parallel radiation and convection, the interaction of incident solar radiation with the earth's surface to produce complex free convection patterns. Hadey et al. [10] studied the flow and heat transfer characteristics of a viscous nanofluid over a nonlinearly stretching sheet in the presence of thermal radiation. Khan et al. [11] analyzed the effects of variable viscosity and thermal conductivity on the flow and heat transfer in a laminar liquid film on a horizontal shrinking/stretching sheet. Khan et al. [12] investigated, by employing the homotopy perturbation transform method (HPTM) and the Padé approximation, the problem of magnetohydrodynamic (MHD) boundary layer flow over a nonlinear porous stretching sheet. Khan et al. [13] considered a two-dimensional, steady magnetohydrodynamic flow and heat transfer analysis of a non-Newtonian fluid in a channel with a constant wall temperature in the presence of thermal radiation.

As the governing equations modelling MHD flow and heat transfer of nanofluids are highly nonlinear, exact solutions are impossible to obtain. Over the years, numerical methods have been developed, improved, and hybred as a way of getting more accurate solutions. The current study seeks to employ a recently developed numerical technique known as spectral relaxation method [14] to solve the problem of magnetohydrodynamic boundary layer flow of nanofluids over a moving surface in the presence of thermal radiation. The current method has been successfully employed in [15-20], among others. We apply this method to the problem of MHD flow of a nanofluid past a stretching sheet in the presence of thermal radiation. The governing boundary layer equations are transformed to a system of nonlinear ordinary differential by using suitable local similarity variables.

\section{Mathematical formulation}

We consider the steady MHD boundary layer flow of a nanofluid past a moving semiinfinite flat plate in a uniform free stream in the presence of thermal radiation. We assume that the velocity of the uniform stream is $U$ and that of the plate is $U_{w}=\lambda U$, where $\lambda$ is the velocity parameter. The flow is assumed to take place at $y \geq 0$, with $y$ being the coordinate measured normal to the moving surface. A uniform magnetic field is applied in the $y$ direction. At moving surface, the temperature and the nanoparticles take constant values $T_{w}$ and $C_{w}$, respectively, while the free stream values are taken to be $T_{\infty}$ and $C_{\infty}$, respectively. Following the nanofluid model proposed by Tiwari and Das [21] along with Boussinesq and boundary layer approximations, the governing equations for the present problem are:

$$
\begin{aligned}
& \frac{\partial u}{\partial x}+\frac{\partial v}{\partial y}=0, \\
& u \frac{\partial u}{\partial x}+v \frac{\partial u}{\partial y}=v \frac{\partial^{2} u}{\partial y^{2}}-\frac{\sigma B_{0}^{2}}{\rho_{f}} u, \\
& u \frac{\partial T}{\partial x}+v \frac{\partial T}{\partial y}=\alpha \frac{\partial^{2} T}{\partial y^{2}}-\frac{\alpha}{k} \frac{\partial q_{r}}{\partial y}+\tau\left[D_{B} \frac{\partial C}{\partial y} \frac{\partial T}{\partial y}+\left(\frac{D_{T}}{T_{\infty}}\right)\left(\frac{\partial T}{\partial y}\right)^{2}\right], \\
& u \frac{\partial C}{\partial x}+v \frac{\partial C}{\partial y}=D_{B} \frac{\partial^{2} C}{\partial y^{2}}+\frac{D_{B}}{T_{\infty}} \frac{\partial^{2} T}{\partial y^{2}}
\end{aligned}
$$


where $u$ and $v$ are the velocity components along the $x$-axis and $y$-axis, respectively, $\alpha=$ $k /(\rho c)_{f}$ is the thermal diffusivity of the fluid, $v$ is the kinematic viscosity coefficient, $k$ is the thermal conductivity, $q_{r}$ is the heat flux, $D_{B}$ is the Brownian diffusion coefficient, $D_{T}$ is the thermophoresis diffusion coefficient, $B_{0}$ is the uniform magnetic field strength of the base fluid, $\sigma$ is the electrical conductivity of the base fluid, $\tau$ is the ratio of the nanoparticle heat capacity and the base fluid heat capacity.

The boundary conditions are taken as

$$
\begin{aligned}
& v=0, \quad u=\lambda U, \quad T=T_{w}, \quad C=C_{w} \quad \text { at } y=0, \\
& u \rightarrow U, \quad T \rightarrow T_{\infty}, \quad C \rightarrow C_{\infty} \quad \text { as } y \rightarrow \infty .
\end{aligned}
$$

The surface moving parameter $\lambda>0$ corresponds to the downstream movement of the plate from the origin, while $\lambda<0$ corresponds to the upstream movement of the plate.

By using the Rosseland diffusion approximation (Hossain et al. [22]) and following Raptis [23] among other researchers, the radiative heat flux $q_{r}$ is given by

$$
q_{r}=-\frac{4 \sigma^{*}}{3 K_{s}} \frac{\partial T^{4}}{\partial y}
$$

where $\sigma^{*}$ and $K_{s}$ are the Stefan-Boltzman constant and the Rosseland mean absorption coefficient, respectively. We assume that the temperature differences within the flow are sufficiently small such that $T^{4}$ may be expressed as a linear function of temperature.

$$
T^{4} \approx 4 T_{\infty}^{3} T-3 T_{\infty}^{4}
$$

Using (7) and (8) in the last term of equation (3), we obtain

$$
\frac{\partial q_{r}}{\partial y}=-\frac{16 \sigma^{*} T_{\infty}^{3}}{3 K_{s}} \frac{\partial^{2} T}{\partial y^{2}}
$$

\subsection{Similarity transformations}

In order to reduce the governing equations into a system of ordinary differential equations, we introduce the following local similarity variables:

$$
\begin{aligned}
& \psi=(2 U \nu x)^{\frac{1}{2}} f(\eta), \quad \theta(\eta)=\frac{T-T_{\infty}}{T_{w}-T_{\infty}}, \\
& \phi(\eta)=\frac{C-C_{\infty}}{C_{w}-C_{\infty}}, \quad \eta=(U / 2 v x)^{\frac{1}{2}} y,
\end{aligned}
$$

where $f(\eta)$ is the dimensionless stream function, $\eta$ is the similarity variable, $\theta(\eta)$ is the dimensionless temperature, $\phi(\eta)$ is the dimensionless nanoparticles concentration. It is worth mentioning that the continuity equation (1) is identically satisfied from our choice of the stream function with $u=\frac{\partial \psi}{\partial y}$ and $v=-\frac{\partial \psi}{\partial x}$. Substituting the similarity variables into equations (2)-(4) gives

$$
\begin{aligned}
& f^{\prime \prime \prime}+\not f^{\prime \prime}-H a f^{\prime}=0, \\
& \left(\frac{3+4 R}{P r}\right) \theta^{\prime \prime}+f \theta^{\prime}+N b \phi^{\prime} \theta^{\prime}+N t \theta^{\prime 2}=0,
\end{aligned}
$$




$$
\phi^{\prime \prime}+\operatorname{Lef} \phi^{\prime}+\frac{N t}{N b} \theta^{\prime \prime}=0
$$

subject to the boundary conditions

$$
\begin{aligned}
& f(0)=0, \quad f^{\prime}(0)=\lambda, \quad \theta(0)=1, \quad \phi(0)=1, \\
& f^{\prime} \rightarrow 1, \quad \theta \rightarrow 0, \quad \phi \rightarrow 0 \quad \text { as } \eta \rightarrow \infty,
\end{aligned}
$$

where $R=\frac{4 \alpha \delta T_{\infty}^{3}}{k k}$ is the radiation parameter, $\operatorname{Pr}=\frac{v}{\alpha}$ is the Prandtl number, $L e=\frac{\nu}{D_{B}}$ is the Lewis number, $H a=\frac{2 \times B_{0}^{2}}{U_{\rho f}}$ is the local Hartman number, $N b=\frac{(\rho c)_{p} D_{B}\left(\phi_{W}-\phi_{\infty}\right)}{(\rho C) f_{v}}$ is the Brownian motion parameter and $N t=\frac{(\rho C)_{\rho} \rho_{T}\left(T_{W}-T_{\infty}\right)}{(\rho c) f T_{\infty} \nu}$ is the thermophoresis parameter.

The quantities of engineering interest are the skin-friction coefficient $C_{f}$, the local Nusselt number $N u_{x}$, and the local Sherwood number $S h_{x}$. These quantities are defined as follows:

$$
C_{f}=\frac{\tau_{w}}{\rho u^{2}}, \quad N u_{x}=\frac{q_{w}}{k\left(T_{w}-T_{\infty}\right)}, \quad S h_{x}=\frac{x q_{m}}{D_{B}\left(C_{w}-C_{\infty}\right)},
$$

where $\tau_{w}, q_{w}$ and $q_{m}$ are the shear stress, heat flux and mass flux at the surface, respectively. Upon using the similarity variables into the above expressions, we get

$$
(2 R e)^{\frac{1}{2}} C_{f}=f^{\prime \prime}(0), \quad\left(\frac{R e_{x}}{2}\right)^{-1} 2 N u_{x}=-\theta^{\prime}(0), \quad\left(\frac{R e_{x}}{2}\right)^{-1} 2 S h_{x}=-\phi^{\prime}(0),
$$

where $R e_{x}=U x / v$ is the local Reynolds number.

\section{Method of solution}

To solve the set of ordinary differential equations (11)-(13) together with the boundary conditions (14) and (15), we employ the Chebyshev pseudo-spectral method known as spectral relaxation method (SRM). This method transforms sets of nonlinear ordinary differential into sets of linear ordinary differential equations. The entire computational procedure is implemented using a program written in MATLAB computer language. The fluid velocity, temperature, the local skin-friction coefficient and the local Nusselt and Sherwood numbers are determined from these numerical computations. The SRM algorithm starts with the assumption of having a system of $m$ nonlinear ordinary differential equations in $m$ unknowns functions, $z_{i}(\eta), i=1,2, \ldots, m$, where $\eta \in[a, b]$ is the independent variable. To solve the resultant iterative scheme, we then use the Chebyshev pseudospectral method. The details of the spectral methods can be found in Canuto et al. [24], Trefethen [25]. Before applying the spectral method, the domain on which the governing equation is defined is transformed to the interval $[-1,1]$ on which the spectral method can then be implemented. We use the transformation $\eta=(b-a)(\tau+1) / 2$ to map the interval $[a, b]$ to $[-1,1]$.

To apply the SRM to the nonlinear ordinary differential equations, we first set $f^{\prime}(\eta)=g(\eta)$ and then write the equations as the following set of equations:

$$
\begin{aligned}
& f^{\prime}=g, \\
& g^{\prime \prime}+f g^{\prime}-g^{2}-H a g=0,
\end{aligned}
$$




$$
\begin{aligned}
& \left(\frac{3+4 R}{P r}\right) \theta^{\prime \prime}+f \theta^{\prime}+N b \theta^{\prime} \phi^{\prime}+N t \theta^{\prime 2}=0, \\
& \phi^{\prime \prime}+\operatorname{Lef} \phi^{\prime}+\frac{N t}{N b} \theta^{\prime \prime}=0,
\end{aligned}
$$

and the boundary conditions become

$$
\begin{array}{ll}
f(0)=0, & g(0)=\lambda, \quad \theta(0)=\phi(0)=1, \\
g(\infty)=1, & \theta(\infty)=0, \quad \phi(\infty)=0 .
\end{array}
$$

In view of the SRM, we obtain the following iterative scheme:

$$
\begin{aligned}
& f_{r+1}^{\prime}=g_{r}, \quad f_{r+1}(0)=0, \\
& g_{r+1}^{\prime \prime}+f_{r+1} g_{r+1}^{\prime}-H a g_{r+1}=0, \quad g_{r+1}(0)=\lambda, \quad g_{r+1}(\infty)=1, \\
& \left(\frac{3+4 R}{P r}\right) \theta_{r+1}^{\prime \prime}+f_{r+1} \theta_{r+1}^{\prime}+N b \theta_{r+1}^{\prime}=-N t_{r+1}^{\prime 2}, \quad \theta_{r+1}(0)=1, \quad \theta_{r+1}(\infty)=0, \\
& \phi_{r+1}^{\prime \prime}+L e f_{r+1} \phi_{r+1}^{\prime}=-\frac{N t}{N b} \theta_{r+1}^{\prime \prime}, \quad \phi_{r+1}(0)=1, \quad \phi_{r+1}(\infty)=0 .
\end{aligned}
$$

The above equations form a system of linear decoupled equations which can be solved iteratively for $r=1,2, \ldots$, starting from initial guesses/approximations $\left(g_{0}(\eta), \theta(\eta), \phi(\eta)\right)$.

Applying the Chebyshev pseudo-spectral method to (23)-(26), we obtain

$$
\begin{array}{lll}
A_{1} \mathbf{f}_{r+1}=B_{1}, & f_{r+1}\left(\tau_{\bar{N}}\right)=0, & \\
A_{2} \mathbf{g}_{r+1}=B_{2}, & g_{r+1}\left(\tau_{\bar{N}}\right)=\lambda, & g_{r+1}\left(\tau_{0}\right)=1, \\
A_{3} \theta_{r+1}=B_{3}, & \theta_{r+1}\left(\tau_{\bar{N}}\right)=1, & \theta_{r+1}\left(\tau_{0}\right)=0, \\
A_{4} \phi_{r+1}=B_{4}, & \phi_{r+1}\left(\tau_{\bar{N}}\right)=1, & \phi_{r+1}\left(\tau_{0}\right)=0,
\end{array}
$$

where

$$
\begin{aligned}
& A_{1}=\mathbf{D}, \quad B_{1}=\mathbf{g}_{\mathbf{r}}, \\
& A_{2}=\mathbf{D}^{2}+\operatorname{diag}\left[\mathbf{f}_{i+1}\right] \mathbf{D}-H a \mathbf{I}, \quad B_{2}=0, \\
& A_{3}=\left(\frac{3+4 R}{P r}\right) \mathbf{D}^{2}+\operatorname{diag}\left[\mathbf{f}_{i+1}+N b\right] \mathbf{D}, \quad B_{3}=-N t \theta_{r+1}^{\prime 2}, \\
& A_{4}=\mathbf{D}^{2}+\operatorname{diag}\left[L e \mathbf{f}_{i+1}\right] \mathbf{D}, \quad B_{4}=-\frac{N t}{N b} \theta_{r+1}^{\prime \prime},
\end{aligned}
$$

where I is the identity matrix of size $(\bar{N}+1) \times(\bar{N}+1), \mathbf{f}, \mathbf{g}, \boldsymbol{\phi}$ and $\boldsymbol{\theta}$ are the values of $f, g, \boldsymbol{\phi}$ and $\theta$, respectively, when evaluated at the grid points. Equations (27)-(30) constitute the SRM scheme. The initial approximation required to start the iterative process is

$$
\begin{aligned}
& g_{0}(\eta)=\lambda-1+\eta+(1-\lambda) e^{-\eta} \\
& \theta_{0}(\eta)=e^{-\eta} \\
& \phi_{0}(\eta)=e^{-\eta}
\end{aligned}
$$


Table 1 Comparison of SRM solutions for $-\theta^{\prime}(0)$ and $-\phi^{\prime}(0)$ against those of the bvp $4 c$ as well as those obtained by Olanrewaju et al. [5] for different values of $\mathrm{Pr}$

\begin{tabular}{lllllll}
\hline $\boldsymbol{P r}$ & {$[\mathbf{5}]$} & $\begin{array}{l}\text { bvp4c } \\
\boldsymbol{\theta}^{\prime}(\mathbf{0})\end{array}$ & Present (SRM) & {$[\mathbf{5}]$} & $\begin{array}{l}\text { bvp4c } \\
-\boldsymbol{\phi}^{\prime}(\mathbf{0})\end{array}$ & Present (SRM) \\
\hline 1 & 0.35300909 & 0.35300909 & 0.35300909 & 0.60037641 & 0.60037641 & 0.60037641 \\
2 & 0.44343465 & 0.44343465 & 0.44343465 & 0.56245704 & 0.56245704 & 0.56245704 \\
6 & 0.59307165 & 0.59307165 & 0.59307165 & 0.481913727 & 0.481913727 & 0.481913727 \\
\hline
\end{tabular}

Table 2 Comparison of SRM solutions for $-\theta^{\prime}(0)$ and $-\phi^{\prime}(0)$ against those of the bvp $4 \mathrm{c}$ as well as those obtained by Olanrewaju et al. [5] for different values of Le

\begin{tabular}{lllllll}
\hline Le & {$[\mathbf{5}]$} & $\begin{array}{l}\text { bvp4c } \\
-\boldsymbol{\theta}^{\prime}(\mathbf{0})\end{array}$ & Present (SRM) & [5] & $\begin{array}{l}\text { bvp4c } \\
-\boldsymbol{\phi}^{\prime}(\mathbf{0})\end{array}$ & Present (SRM) \\
\hline 10 & 0.31104609 & 0.31104594 & 0.31104594 & 1.22044717 & 1.22044716 & 1.22044716 \\
20 & 0.31066665 & 0.31066665 & 0.31066665 & 1.6237386 & 1.62037385 & 1.62037385 \\
50 & 0.310302944 & 0.31030279 & 0.31030279 & 2.36541737 & 2.36541736 & 2.36541736 \\
\hline
\end{tabular}

which are randomly chosen functions that satisfy the boundary conditions. The iteration is repeated until convergence is achieved. The convergence of the SRM scheme is defined in terms of the infinity norm as

$$
E r=\operatorname{Max}\left(\left\|f_{r+1}-f_{r}\right\| ;\left\|g_{r+1}-g_{r}\right\| ;\left\|\theta_{r+1}-\theta_{r}\right\| ;\left\|\phi_{r+1}-\phi_{r}\right\|\right) .
$$

Accuracy of the scheme is established by increasing the number of collocation points $N$ until the solutions are consistent and further increases do not change the value of the solutions.

\section{Results and discussion}

The system of ordinary differential equations (12)-(13) subject to the boundary conditions (14)-(15) are numerically solved by using spectral relaxation method (SRM). This is a recently developed method, and details of the method are found in [26]. The SRM results presented in this work were obtained using $N=50$ collocation points, and also the convergence was achieved after as few as five iterations. We also take 15 to be the infinity value $\eta_{\infty}$. We use these default values for the parameters $\operatorname{Pr}=0.71, N t=N b=0.3, L e=2$, $R=2, \lambda=0.1$.

Tables 1 through 5 show a comparison among the SRM results to those previously obtained results as well as the bvp $4 \mathrm{c}$ results. As can be clearly observed from these tables, there are excellent agreements in all the tables giving us confidence in the findings of this study. Table 1 depicts the effects of increasing the values of the Prandtl number on heat and mass transfers. Physically, the Prandtl number is a dimensionless number which is the ratio of momentum diffusivity (kinematic viscosity) to thermal diffusivity. Increasing values of the Prandtl number means that momentum diffusivity dominates thermal diffusivity. Thus, the rate of heat transfer at the surface increases with increasing values of the Prandtl number while the rate of mass transfer is reduced as the Prandtl number increases. Table 2 shows the effect of increasing the Lewis number $L e$ on the heat and mass transfer coefficients. The Lewis number is defined as the ratio of thermal diffusivity to mass diffusivity or the ratio of the Schmidt number to the Prandtl number. As can be clearly seen in Table 2, increasing the Lewis number means that the fluid becomes more 
Table 3 Comparison of SRM solutions for $-\theta^{\prime}(0)$ and $-\phi^{\prime}(0)$ against those of the bvp $4 \mathrm{c}$ as well as those obtained by Olanrewaju et al. [5] for different values of $\lambda$

\begin{tabular}{lllllll}
\hline $\boldsymbol{\lambda}$ & {$[5]$} & $\begin{array}{l}\text { bvp4c } \\
\boldsymbol{\theta}^{\prime}(\mathbf{0})\end{array}$ & Present (SRM) & [5] & $\begin{array}{l}\text { bvp4c } \\
-\boldsymbol{\phi}^{\prime}(\mathbf{0})\end{array}$ & Present (SRM) \\
\hline 0.5 & 0.36940306 & 0.36940302 & 0.36940302 & 0.81838542 & 0.81838541 & 0.81838541 \\
1 & 0.42412772 & 0.42412771 & 0.42412771 & 1.0624088 & 1.0624088 & 1.0624088 \\
2 & 0.50948651 & 0.50948651 & 0.50948651 & 1.32551731 & 1.32551731 & 1.32551731 \\
\hline
\end{tabular}

Table 4 Computations of $-\theta^{\prime}(0)$ and $-\phi^{\prime}(0)$ at different values of $N b$ and $N t$

\begin{tabular}{lll}
\hline $\boldsymbol{N b}$ & $\boldsymbol{- \theta}^{\prime}(\mathbf{0})$ & $-\boldsymbol{\phi}^{\prime}(\mathbf{0})$ \\
\hline 0.1 & 0.27455519 & 0.48306117 \\
0.5 & 0.24471306 & 0.53071472 \\
1 & 0.21121513 & 0.53632970 \\
\hline & & \\
\hline $\boldsymbol{N t}$ & $-\boldsymbol{\theta}^{\prime}(\mathbf{0})$ & $-\boldsymbol{\phi}^{\prime}(\mathbf{0})$ \\
\hline 0.1 & 0.27455519 & 0.48306117 \\
0.5 & 0.25536198 & 0.32985814 \\
1 & 0.23358969 & 0.25949965 \\
\hline
\end{tabular}

Table 5 Comparison of SRM solutions for $-f^{\prime \prime}(0)$ against those of the bvp $4 \mathrm{c}$ for different values of $\lambda$

\begin{tabular}{lll}
\hline $\boldsymbol{\lambda}$ & $\begin{array}{l}\text { bvp4c } \\
\boldsymbol{f}^{\prime \prime}(\mathbf{0})\end{array}$ & Present (SRM) \\
\hline 0.1 & 0.46251223 & 0.46251223 \\
0.2 & 0.44315478 & 0.44315478 \\
0.4 & 0.32874112 & 0.32874112 \\
\hline
\end{tabular}

viscous therefore causing increases in the rate of mass transfer at the surface. As expected, increasing the Lewis number reduces the rate of heat transfer on the surface as this corresponds to the reduced Prandtl number. In Table 3, we display the effect of increasing the values of the moving surface parameter $\lambda$ on the rates of heat and mass transfer. These rates both increase with increasing values of the surface moving parameter. Table 4 depicts the effects of Brownian motion parameter $N b$ and the thermophoresis parameter on the heat and mass transfer coefficients. The rate of heat transfer on the surface as expected is reduced as the Brownian motion parameter increases, while the rate of mass transfer slightly increases as this parameter increases. Also in Table 4, we depict the effect of the thermophoresis parameter Nt on the Nusselt and Sherwood numbers. By definition thermophoresis is the migration of a colloidal particle in a solution in response to a macroscopic temperature gradient. Both the Nusselt number and the Sherwood number are reduced as the values of this parameter increases.

In Table 5 we show the influence of the velocity parameter on the skin friction. Increasing positive values of $\lambda$ corresponds to accelerated movement of the plate. This in turn results in reduction of skin friction. The effects of physical parameters of importance in this study on the velocity, temperature and nanoparticles distributions are depicted in Figures 1-9. Figure 1 displays the effect of the Hartman number $\mathrm{Ha}$ on the dimensionless velocity. The presence of a magnetic field normal to the flow in an electrically conducting fluid gives rise to a Lorentz force, which acts against the flow. Thus the velocity distributions of the nanofluid are greatly reduced with increasing values of the Hartman number. 
Figure 1 Graph of the SRM solutions of the velocity profiles for different values of $\mathrm{Ha}$.

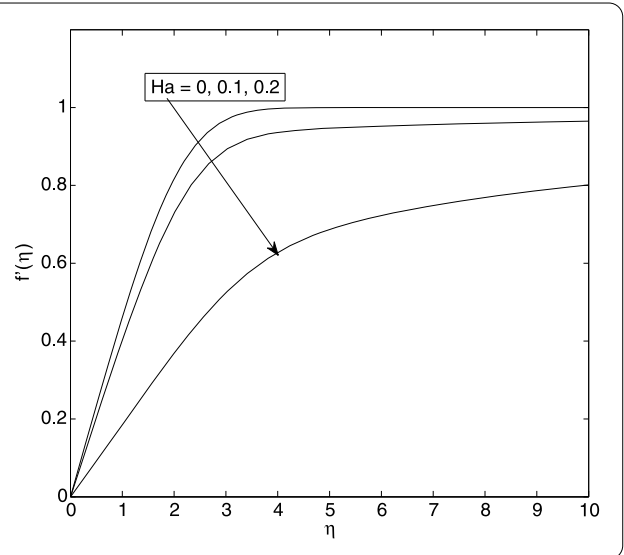

Figure 2 Graph of the SRM solutions of the velocity profiles for different values of $\lambda$.

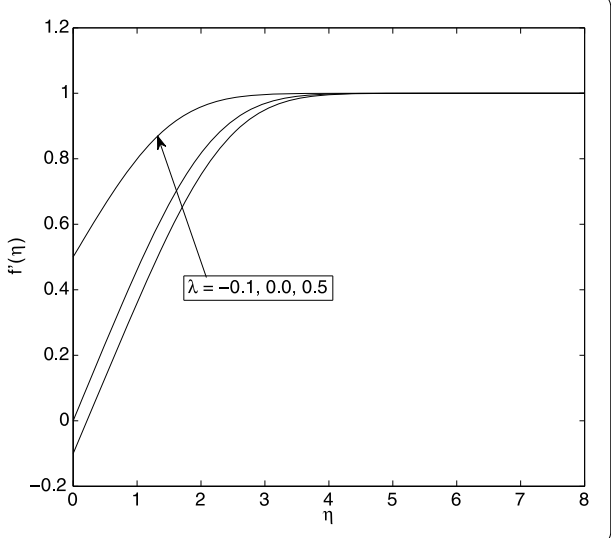

Figure 3 Temperature profiles for different values of $\mathrm{Ha}$.

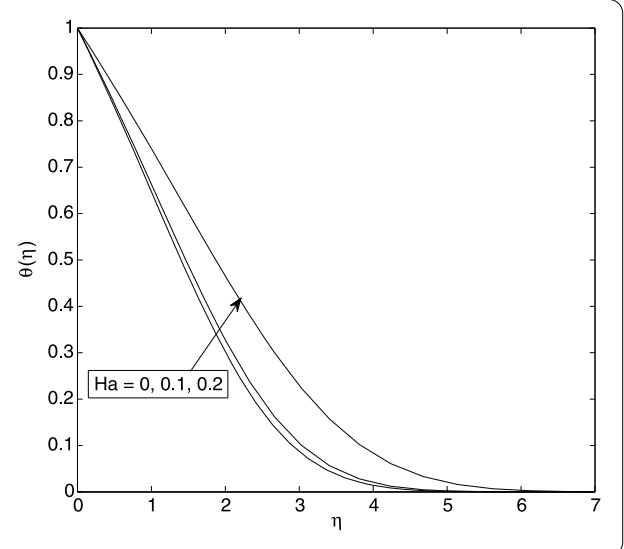

Figure 2 presents the effect of $\lambda$ on the velocity profiles. Increasing the down stream movement of the plate from the origin, as expected, enhances the fluid velocity. The influence of the magnetic field parameter on the temperature distribution is shown in Figure 3. From this figure, we observe that the temperature profiles increase with increasing values of the magnetic field parameter. This implies that the applied magnetic field tends to heat the fluid, thus reducing the heat transfer from the wall.

Figure 4 shows the effect of increasing the radiation parameter $R$ on the temperature distribution. We observe in this figure that the thickness of the thermal boundary layer 


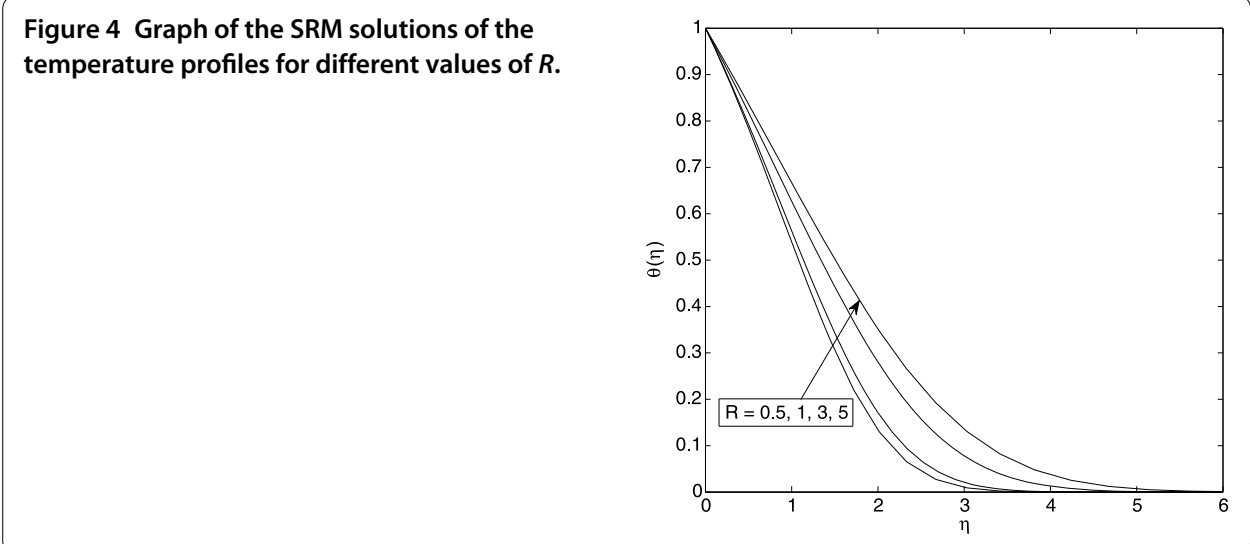

Figure 5 Influence of $\mathrm{Nt}$ on the temperature profiles.

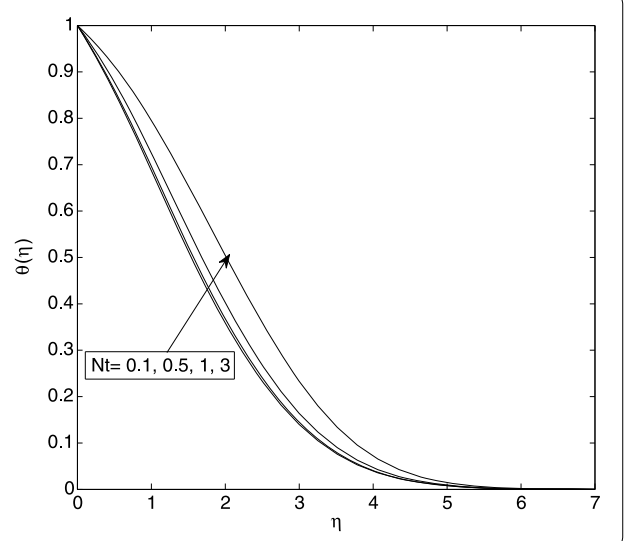

Figure 6 Graph of the SRM solutions of the temperature profiles for different values of $N b$.

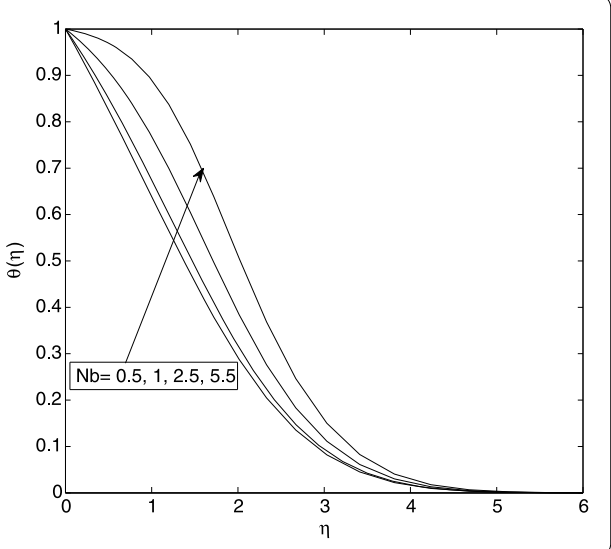

increases as the values of the radiation parameter are increased. Thus thermal radiation enhances thermal diffusion.

In Figure 5, we have the influence of the thermophoresis parameter on the temperature distribution. Increases in this parameter physically imply high temperature gradients. This in turn results in high temperature distributions within the nanofluid flow. Increasing the values of the Brownian motion parameter $\mathrm{Nb}$ results in thickening of the thermal boundary layer. Thus enhancing the temperature of the nanofluid as can be easily seen in Figure 6. 
Figure 7 Graph of the SRM solutions of the nanoparticle volume fraction profiles for different values of $\mathrm{Ha}$.

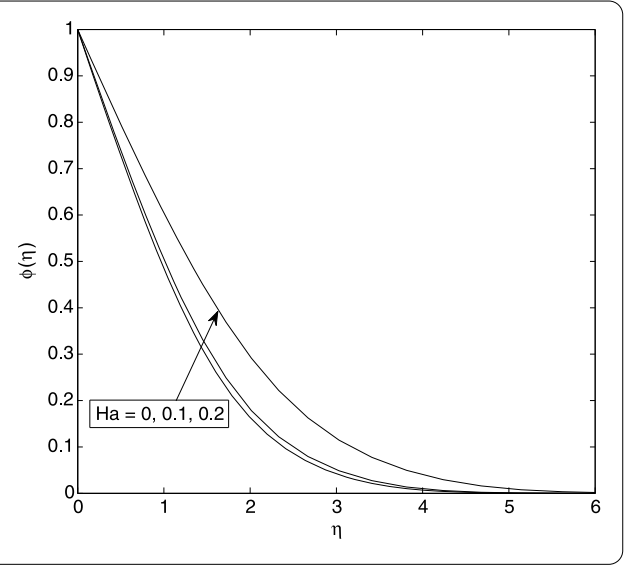

Figure 8 Influence of Le on the nanoparticle volume fraction profiles.

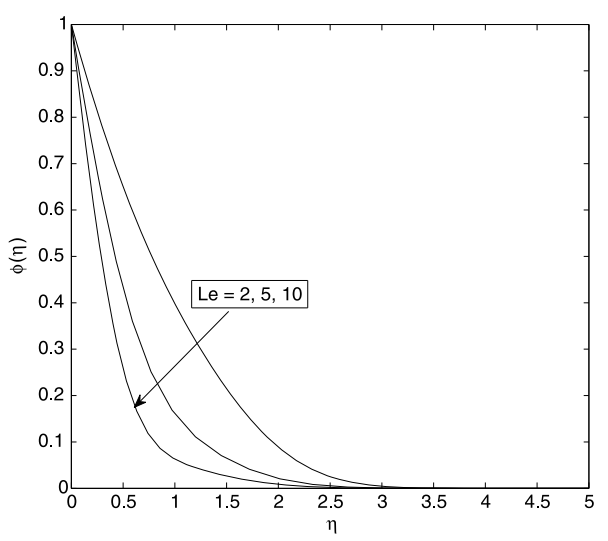

Figure 9 Influence of $\mathrm{Nt}$ on the nanoparticle volume fraction profiles.

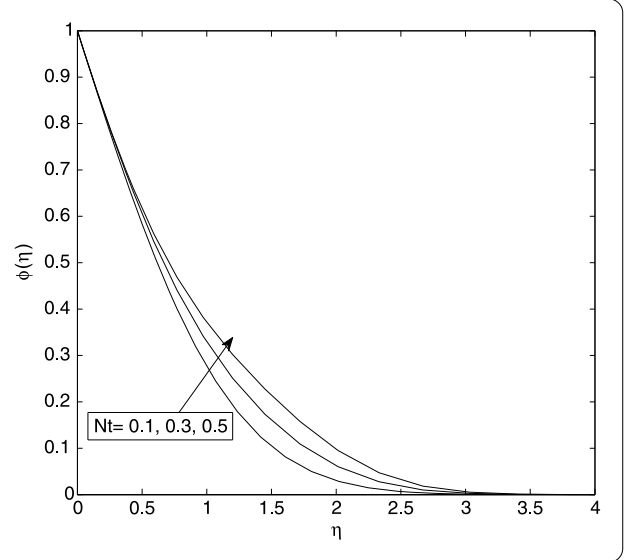

The effect of the magnetic field strength $\mathrm{Ha}$ on the nanoparticle volume fraction profiles is shown in Figure 7. Application of a normal magnetic field results in slowing down of the nanofluid flow thereby resulting in volumetric increase of the nanoparticles within the flow as less particles are now transported per given time.

In Figure 8 we depict the effect of the Lewis number on the nanoparticles. Large values of the Lewis number implies increased values of the Schmidt number which in turn results in thinning of the solutal boundary layer as can be clearly seen in Figure 8. Increasing 
the thermophoresis as expected results in increase of the nanoparticle volume fraction profiles. This is displayed in Figure 9.

\section{Conclusion}

Numerical analysis has been carried out on MHD boundary layer flow of nanofluids over a moving surface in the presence of thermal radiation. The governing partial differential equations were transformed into a system of ordinary differential equations using suitable similarity transformations. The resultant equations were then solved using the spectral relaxation method. The accuracy of the SRM is validated against the MATLAB in-built bvp $4 \mathrm{c}$ routine for solving boundary value problems as well as previously obtained results. An excellent agreement was observed between our results and those obtained using other methods giving confidence to our present results. We observed that the local temperature rises as the Brownian motion, thermophoresis and radiation effects intensify. Lastly, the Nusselt number decreases while the Sherwood number increases as the Brownian motion and thermophoresis effects are increased.

\footnotetext{
Competing interests

The authors declare that they have no competing interests.
}

Authors' contributions

SS formulated, generated and discussed the results, and JP discussed the results and proofread the manuscript. All authors read and approved the final manuscript.

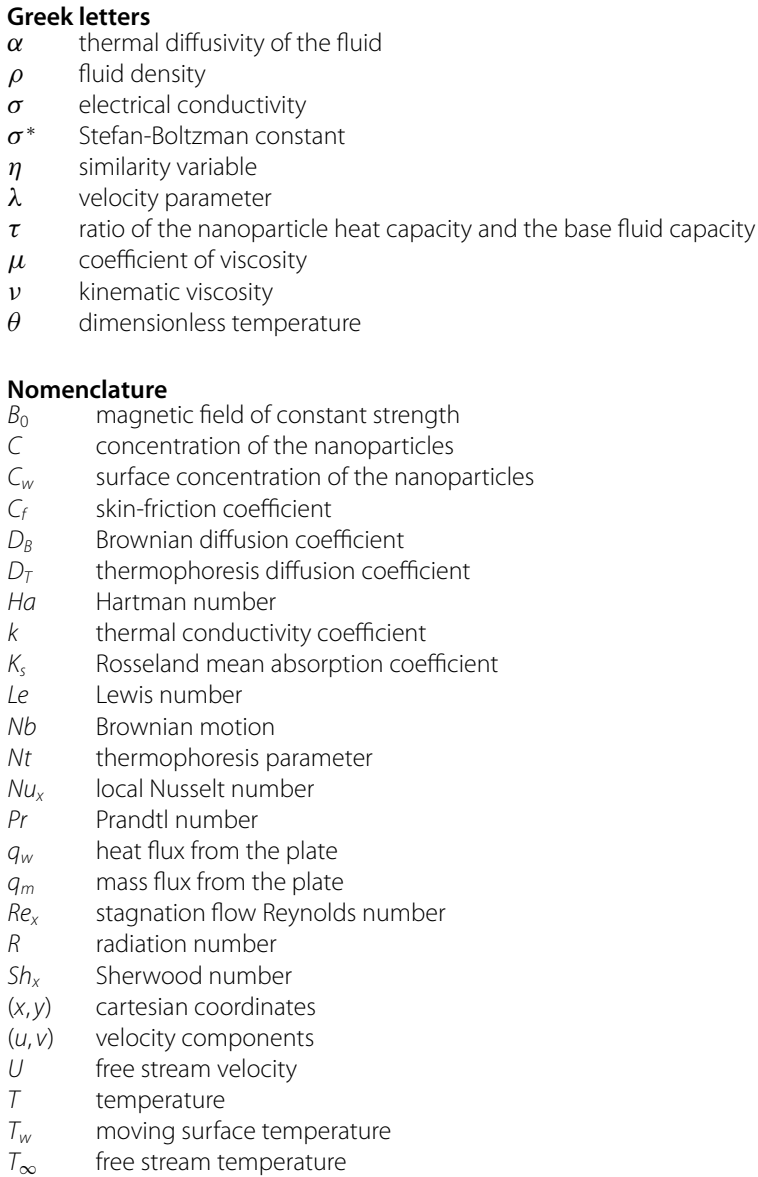




\section{Author details}

${ }^{1}$ Department of Mathematics, University of Venda, Private Bag X5050, Thohoyandou, 0950, South Africa. ${ }^{2}$ Department of Mathematics, University of Botswana, Private Bag UB 0022, Gaborone, Botswana.

\section{Acknowledgements}

The authors wish to acknowledge financial support from the University of Venda, NRF and University of Botswana.

Received: 19 August 2013 Accepted: 1 December 2013 Published: 02 Jan 2014

\section{References}

1. Choi, SUS: Enhancing thermal conductivity of fluids with nanoparticles. In: Developments and Applications of Non-Newtonian Flows. FED, vol. 231/MD, vol. 66, pp. 99-105 (1995)

2. Mnyusiwalla, A, Daar, AS, Singer, PA: Mind the gap: science and ethics in nanotechnology. Nanotechnology 14 R9-R13 (2003)

3. Kakaç, S, Pramuanjaroenkij, A: Review of convective heat transfer enhancement with nanofluids. Int. J. Heat Mass Transf. 52, 3187-3196 (2009)

4. Godson, L, Raja, B, Lal, DM, Wongwises, S: Enhancement of heat transfer using nanofluid - An overview. Renew. Sustain. Energy Rev. 14, 629-641 (2010)

5. Olanrewaju, PO, Olanrewaju, MA, Adesanya, AO: Boundary layer flow of nanofluids over a moving surface in a flowing fluid in the presence of radiation. Int. J. Appl. Sci. Technol. 2(1), 274-285 (2012)

6. Gbadeyan, JA, Olanrewaju, MA, Olanrewaju, PO: Boundary layer flow of a nanofluid past a stretching sheet with a convective boundary condition in the presence of magnetic field and thermal radiation. Aust. J. Basic Appl. Sci. 5(9), 1323-1334 (2011)

7. Khan, WA, Aziz, A: Natural convection flow of a nanofluid over a vertical plate with uniform surface heat flux. Int. J. Therm. Sci. 50, 1207-1214 (2011)

8. Makinde, OD, Aziz, A: Boundary layer flow of a nanofluid past a stretching sheet with a convective boundary condition. Int. J. Therm. Sci. 50, 1326-1332 (2011)

9. Khan, MS, Karim, I, Ali, LE, Islam, A: Unsteady MHD free convection boundary-layer flow of a nanofluid along a stretching sheet with thermal radiation and viscous dissipation effects. Int. Nano Lett. 2, 24 (2012). doi:10.1186/2228-5326-2-24

10. Hady, FM, Ibrahim, FS, Abdel-Gaied, SM, Eid, MR: Radiation effect on viscous flow of a nanofluid and heat transfer over a nonlinearly stretching sheet. Nanoscale Res. Lett. 7, 229-242 (2012)

11. Khan, Y, Wua, Q, Faraz, N, Yildirim, A: The effects of variable viscosity and thermal conductivity on a thin film flow ove a shrinking/stretching sheet. Comput. Math. Appl. 61(11), 3391-3399 (2011)

12. Khan, Y, Abdou, MA, Faraz, N, Yildirim, A, Wu, Q: Numerical solution of MHD flow over a nonlinear porous stretching sheet. Iran. J. Chem. Chem. Eng. 31(3), 125-132 (2012)

13. Khan, Y, Wu, Q, Faraz, N, Yildirim, A, Mohyud-Din, ST: Heat transfer analysis on the magnetohydrodynamic flow of a non-Newtonian fluid in the presence of thermal radiation: an analytic solution. Z. Naturforsch. A 67a, 147-152 (2012)

14. Motsa, SS, Dlamini, P, Khumalo, M: A new multistage spectral relaxation method for solving chaotic initial value systems. Nonlinear Dyn. 72, 265-283 (2013)

15. Motsa, SS, Dlamini, PG, Khumalo, M: Solving hyperchaotic systems using the spectral relaxation method. Abstr. Appl. Anal. (2012). doi:10.1155/2012/203461

16. Motsa, SS, Dlamini, P, Khumalo, M: A new multistage spectral relaxation method for solving chaotic initial value systems. Nonlinear Dyn. (2013). doi:10.1007/s11071-012-0712-8

17. Motsa, SS, Makukula, ZG: On spectral relaxation method approach for steady von Kármán flow of a Reiner-Rivlin fluid with Joule heating, viscous dissipation and suction/injection. Cent. Eur. J. Phys. (2013). doi:10.2478/s11534-013-0182-8

18. Shateyi, S, Makinde, OD: Hydromagnetic stagnation-point flow towards a radially stretching convectively heated disk Math. Probl. Eng. (2013). doi:10.1155/2013/616947

19. Shateyi, S, Marewo, GT: A new numerical approach of MHD flow with heat and mass transfer for the UCM fluid over a stretching surface in the presence of thermal radiation. Math. Probl. Eng. (2013). doi:10.1155/2013/670205

20. Shateyi, S: A new numerical approach for MHD flow of a Maxwell fluid past a vertical stretching sheet in the presence of thermophoresis and chemical reaction. Bound. Value Probl. (2013). doi:10.1186/1687-2770-2013-196

21. Tiwari, RK, Das, MK: Heat transfer augmentation in a two-sided lid-driven differentially heated square cavity utilizing nanofluids. Int. J. Heat Mass Transf. 50, 2002-2018 (2007)

22. Hossain, MA, Takhar, HS: Radiation effect on mixed convection along a vertical plate with uniform surface temperature. Heat Mass Transf. 31, 243-248 (1996)

23. Raptis, A: Flow of a micropolar fluid past a continuously moving plate by the presence of radiation. Int. J. Heat Mass Transf. 41, 2865-2866 (1998)

24. Canuto, C, Hussaini, MY, Quarteroni, A, Zang, TA: Spectral Methods in Fluid Dynamics. Springer, Berlin (1988)

25. Trefethen, LN: Spectral Methods in MATLAB. SIAM, Philadelphia (2000)

26. Motsa, SS: A new spectral relaxation method for similarity variable nonlinear boundary layer flow systems. Chem. Eng. Commun. (2013). doi:10.1080/00986445.2013.766882 